Ann. Zootech., I977, 26 (3), 345-353.

\title{
Différences intraraces de réponse ovarienne \\ à un traitement de superovulation par PMSG chez des vaches de race charolaise. Fréquence des naissances gémellaires
}

\author{
J. C. MARIANA *, P. GIRARD et D. CHUPIN * \\ avec la collaboration technique de $\mathrm{Y}$. de FonTAubert \\ * Station de Physiologie de la Reproduction, \\ Centre de Recherches de Tours, I.N.R.A., \\ Noutilly, 37380 Monnaie (France)
}

\begin{abstract}
Résumé
6 lignées de demi-søeurs de race Charolaise ont été testées sur leur taux de superovulation et leur aptitude à produire des naissances gémellaires en réponse à une injection de I ooo UI de PMSG le 16 e jour d'un cycle normal.

Le taux de superovulation varie de 32,5 à 75 , o p. too et le nombre moyen d'ovulation de I,47 à 3,62. L'efficacité de la superovulation (nombre de veaux nés pour ioo ovules pondus) varie avec le taux d'ovulation mais également entre lignées (de 12 à $60 \%$ ). Cette variation reflète en partie des différences du taux de gestation (de 31,5 à $66,6 \%$ pour les mêmes groupes).

Au total, la productivité numérique (nombre de veaux nés vivants pour ioo femelles mises en reproduction) diffère selon les lignées. L'aptitude à la superovulation ne suffit pas pour expliquer ces différences puisque le meilleur résultat ( I I \%) est obtenu avec la lignée qui présente la plus faible réponse ovarienne (I 47 ovules pour 100 vaches).
\end{abstract}

\section{Introduction}

La réponse ovarienne estimée par le nombre moyen d'ovulations, obtenue après un traitement de superovulation par PMSG varie selon les races (MAULEON et al., I970; TURMAN et al., I97I; MENissier et al., I974). A l'intérieur d'une race donnée (FFPN) pour un lot de femelles homogène, on observe une variabilité individuelle importante du taux d'ovulation (MARIANa et al., I970). Cette réponse se réaliserait par seuils successifs (MARIANA, r970), seuils qui, pour un individu donné, varieraient dans le temps : il est difficile de mettre en évidence une répétabilité de la réponse à deux traitements successifs quel que soit l'intervalle entre ceux-ci. Par contre, à l'échelle de la population la distribution des réponses ovariennes est répétable, ce qui semble indiquer une constance de la distribution des seuils (MARIANA, I970). 
La variabilité individuelle de réponse ovarienne à PMSG pourrait donc présenter une composante génétique. Ainsi, des rattes de trois souches sélectionnées sur leur sensibilité à FSH répondent différemment à PMSG (MARIANA, I974). De plus, lorsque la dose de PMSG augmente, la variance de la moyenne augmente aussi, en partie du fait de l'augmentation de la moyenne, et en partie du fait d'une meilleure manifestation des différences individuelles de sensibilité ovarienne (MARIANA, I974). Chez le Hamster, MoORE et Greenwald (I974) n'observent des variations saisonnières du taux d'ovulation qu'en réponse à des doses fortes de PMSG. A dose faible, la réponse ne varie pas.

L'étude présentée a pour objet de comparer les différences de sensibilité à PMSG chez les bovins en fonction de l'origine paternelle.

\section{Matériel et méthodes}

Cette étude porte sur 59 vaches allaitantes de race Charolaise provenant d'une station de testage sur les qualités d'élevage et réparties en 6 groupes de demisours de même père. Le nombre de femelles par groupe varie de 5 à I 2 .

La reproduction de ces animaux a été étudiée depuis l'âge de 8 mois jusqu'après leur $4^{\mathrm{e}}$ vélage inclus. Les deux premières gestations ont été obtenues sans traitement, les deux dernières après un traitement de superovulation. Pendant toute la durée de cette étude, les femelles étaient mises en présence d'un taureau vasectomisé deux fois par jour pour détecter l'œstrus.

TABLEAU I

Taux de superovulation et nombre moyen d'ovulations

Superovulation rate and mean number of ovulations

\begin{tabular}{|c|c|c|c|c|c|}
\hline \multirow{2}{*}{$\begin{array}{l}\text { Groupes } \\
\text { (Groups) }\end{array}$} & \multirow{2}{*}{$\begin{array}{c}\text { Nombre } \\
\text { de femelles } \\
\text { (Number of } \\
\text { females) }\end{array}$} & \multicolumn{3}{|c|}{$\begin{array}{l}\text { p. Ioo de femelles ayant } \\
(p . \text { Ioo of females with })\end{array}$} & \multirow{2}{*}{$\begin{array}{l}\text { Nombre moyen } \\
\text { d'ovulations } \\
\text { (Mean number } \\
\text { of ovulations) } \\
(\sigma)\end{array}$} \\
\hline & & $\begin{array}{c}2 \text { et } 3 \\
\text { ovulations } \\
(2 \text { and } \mathbf{3} \\
\text { ovulations) }\end{array}$ & $\begin{array}{l}\geqslant 4 \text { ovulations } \\
\geqslant(4 \text { ovulations })\end{array}$ & $\begin{array}{c}2 \text { ovulations } \\
\text { et }+ \\
(2 \text { ovulations } \\
\text { and }+)\end{array}$ & \\
\hline I & $2 \mathrm{I}$ & 23,8 & 9,7 & 33,5 & $1,7 I \pm 1,27$ \\
\hline 2 & 8 & 37,5 & 37,5 & $75, \mathrm{o}$ & $3,62 \pm 2,64$ \\
\hline 3 & 19 & 26,3 & 5,3 & 31,6 & $1,47 \pm 0,94$ \\
\hline 5 & 22 & $3 \mathrm{I}, 8$ & 18,2 & 50,0 & $2,00 \pm 1,66$ \\
\hline 6 & 22 & 27,3 & 4,5 & $3 r, 8$ & $\mathbf{1}, 5^{\circ} \pm 0,95$ \\
\hline 9 & I 6 & I 8,7 & 25,0 & 43.7 & $2,25 \pm 1,82$ \\
\hline
\end{tabular}

Les traitements de superovulation ont consisté en l'injection de I ooo UI

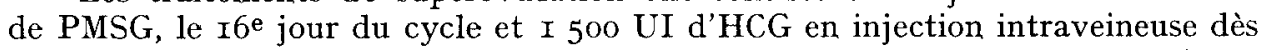
le début de l'œstrus suivant. Le nombre d'ovulations obtenues en réponse à ces traitements de superovulation a été déterminé par endoscopies, ro jours après l'œstrus (MARIANA, Ig69).

Les inséminations ont été réalisées avec de la semence de taureaux Charolais 
donnant des veaux présentant à la naissance un poids faible, pour éviter les difficultés de vélage. La période d'insémination se terminait le ${ }_{5} 5$ août, quelle que soit la date du vélage précédent : chaque année quelques vaches n'ont été inséminées que deux fois, voire une seule fois; certaines n'ont pas été inséminées et ne sont pas incluses dans les dépouillements.

\section{TABLEAU 2}

Taux de gestation et production de veaux après insémination à l'ostrus de traitement Pregnancy rate and production of calves after insemination at the oestrus of treatment

\begin{tabular}{|c|c|c|c|c|}
\hline \multirow{2}{*}{$\begin{array}{l}\text { Groupes } \\
\text { (Groups) }\end{array}$} & \multirow{2}{*}{$\begin{array}{l}\text { Nombre } \\
\text { d'ovules } \\
\text { pondus } \\
\text { (Number } \\
\text { of ova) }\end{array}$} & \multicolumn{3}{|c|}{$\begin{array}{l}\text { Pour roo vaches mises en traitement et inséminées une fois } \\
\text { (For roo cows in treatment and inseminated once) }\end{array}$} \\
\hline & & $\begin{array}{l}\text { Nombre de mises bas } \\
(\text { Number of calvings) }\end{array}$ & $\begin{array}{l}\text { Nombre de veaux nés } \\
(\text { Number of calves born })\end{array} \mid$ & $\begin{array}{l}\text { Nombre de veaux vivants } \\
\text { (Number of calves alive) }\end{array}$ \\
\hline I & I $7 \mathrm{I}$ & 31,5 & 36,8 & 36,8 \\
\hline 2 & $3^{62}$ & 33,3 & 44,3 & 33,3 \\
\hline 3 & 147 & $42, I$ & 68,6 & $63, I$ \\
\hline 5 & 200 & 45,0 & 49,9 & $4^{\circ}, 0$ \\
\hline 6 & 150 & 66,6 & 90,6 & 66,6 \\
\hline 9 & 225 & 40,0 & $4^{6,8}$ & 33,2 \\
\hline
\end{tabular}

TABLEAU 3

Nombre de veaux nés pour roo ovules pondus - Differences entre lignées Number of calves born for Ioo ova - Differences between groups

\begin{tabular}{c|c|c}
\hline \hline $\begin{array}{c}\text { Groupes } \\
\text { (Groups) }\end{array}$ & $\begin{array}{c}\text { Nombre de veaux nés } \\
(\text { Number of calves born) }\end{array}$ & $\begin{array}{l}\text { Nombre de veaux vivants } \\
\text { (Number of calves alive })\end{array}$ \\
\hline & & \\
I & $2 \mathrm{I}$ & $2 \mathrm{I}$ \\
2 & $\mathrm{~N}$ & 9 \\
3 & 46 & 43 \\
5 & 25 & 20 \\
6 & 60 & 44 \\
9 & $2 \mathrm{I}$ & 15 \\
\hline
\end{tabular}

Les différences entre femelles ont été analysées à trois niveaux :

- La réponse ovarienne estimée soit par le pourcentage de femelles ayant pondu deux ovules ou plus, soit par le nombre moyen d'ovules pondus (tabl. I).

- La récolte en veaux produite par le traitement, estimée par le taux de mises-bas après insémination à l'œstrus de traitement et le nombre de veaux nés ou vivants à ces mises-bas (tabl. 2). Une relation a été établie entre le nombre de veaux nés pour roo ovules pondus, en fonction des groupes de femelles (tabl. 3) ou du nombre d'ovulations par femelle (tabl. 4). 


\section{TABLEAU 4}

Nombre de veaux nés pour too ovules pondus - Influence du nombre d'ovules pondus Number of calves born for Ioo ova - Influence of the number of ova

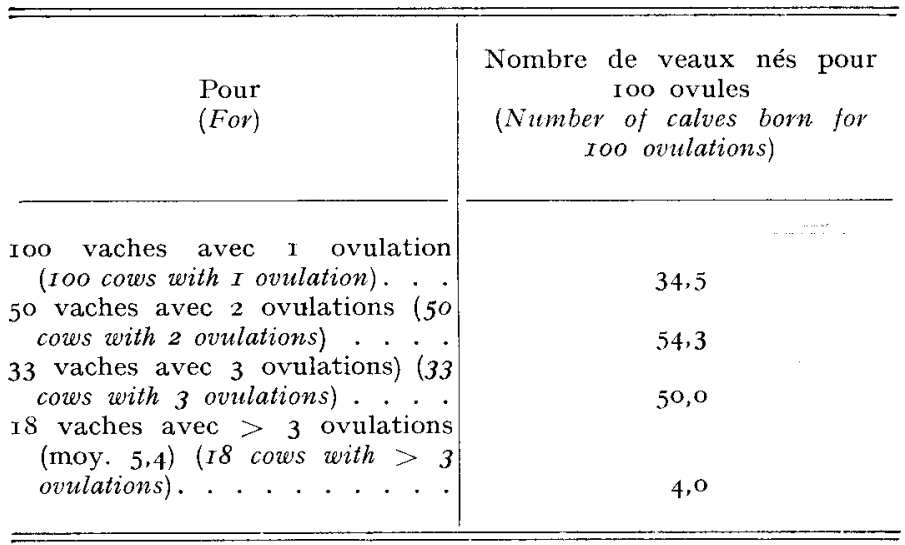

TABLEAU 5

Bilan des vélages en conduite normale (vélages $I$ et 2 ) et avec traitement de superovulation (vélages 3 et 4)

Results of calvings under normal management (calvings $I$ and 2 ) and after superovulation treatment (calvings 3 and 4 )

\begin{tabular}{|c|c|c|c|c|c|c|}
\hline \multirow{2}{*}{$\begin{array}{l}\text { Groupes } \\
\text { (Groups) }\end{array}$} & \multicolumn{3}{|c|}{$\begin{array}{c}\text { Sans traitement } \\
\text { (Without treatment) }\end{array}$} & \multicolumn{3}{|c|}{$\begin{array}{l}\text { Avec traitement. } \\
\text { (With treatment) }\end{array}$} \\
\hline & $\begin{array}{c}\text { p. Ioo } \\
\text { gestantes } \\
(p . \text { Ioo } \\
\text { pregnant })\end{array}$ & $\begin{array}{l}\text { P. Ioo veaux } \\
\text { nés } \\
(p . \text { Ioo calves } \\
\text { born })\end{array}$ & $\left\{\begin{array}{c}\text { p. Ioo veaux } \\
\text { vivants } \\
(p . \text { Ioo calves } \\
\text { alive })\end{array}\right.$ & $\begin{array}{c}\text { p. Ioo } \\
\text { gestantes } \\
(p . \text { Ioo } \\
\text { pregnant })\end{array}$ & $\left|\begin{array}{c}\text { p. Ioo veaux } \\
\text { nés } \\
(p . \text { Ioo calves } \\
\text { born })\end{array}\right|$ & $\begin{array}{c}\text { p. Ioo veaux } \\
\text { vivants } \\
(p . \text { Ioo calves } \\
\text { alive })\end{array}$ \\
\hline I & $9 \mathrm{I}$ & $9 \mathrm{I}$ & 77 & 84 & IOI & 91 \\
\hline 2 & 80 & 80 & 80 & 89 & 127 & IOI \\
\hline 3 & IOO & 100 & 95 & 95 & $\mathrm{I} 2 \mathrm{I}$ & I I I \\
\hline 5 & 96 & 96 & 88 & 90 & I I 2 & 79 \\
\hline 6 & 100 & 100 & 96 & 91 & I I 5 & 91 \\
\hline 9 & 94 & 94 & 94 & 80 & 87 & 74 \\
\hline
\end{tabular}

p. Ioo rapportés au nombre de vaches mises en reproduction ( $p$. Ioo relatively to the number of cows inseminated).

- La récolte en veaux totale par campagne de reproduction en comparaison avec les résultats obtenus pour les $\mathbf{I}^{\mathrm{er}}$ et $2^{\mathrm{e}}$ vélages sans traitements de superovulation (tabl. 5). 


\section{Résultats}

\section{I. - Nombre d'ovulations}

Dans le tableau I, est porté pour chaque groupe de demi-sœurs le taux de réponses supérieures ou égales à 2 ovulations et le nombre moyen d'ovules. Le premier varie de $31,6 \mathrm{p}$. Ioo (groupe 3 ) à $75 \mathrm{p}$. Ioo (groupe 2) et le second paramètre varie de 1,47 (groupe 3 ) à 3,62 (groupe 2) - (différences non significatives).

\section{2. - Taux de gestation - Nombre de veaux nés ou vivants}

Le taux de gestation après insémination à l'œstrus de traitement varie de $3 \mathrm{I}, 5 \mathrm{p}$. Ioo pour le groupe I à $66,6 \mathrm{p}$. Ioo pour le groupe $6(\mathrm{P}<0,05)$ (tabl. 2). Le taux de fécondité (nombre de veaux nés) varie de façon parallèle entre les groupes de demi sours de 36,8 veaux pour cent vaches dans le groupe I à 90,6 dans le groupe 6 .

\section{3. - Efficacité de la superovulation}

Celle-ci est estimée par le nombre de veaux nés pour roo ovules pondus. Entre groupes de demi sours les valeurs de ce paramètre varient de I2 (groupe 2 ) à $46 \mathrm{p}$. Ioo (groupe 3 ) et $60 \mathrm{p}$. Ioo (groupe 6) (différences groupe $2-$ groupe $3 ; \mathrm{P}<0,05$; groupe 2, groupe $6, \mathrm{P}<\mathrm{O}, \mathrm{OI})$. Dans le tableau 4, l'efficacité de la superovulation est calculée en fonction du nombre d'ovulation par femelle. Elle est élevée pour 2 ou 3 ovulations $(54,3$ p. Ioo) et très faible au-dessus (4,0 p. Ioo).

\section{4. - Bilan de reproduction - Productivité numérique}

Dans le tableau 5 sont portés les taux cumulés de vaches fécondées pendant la période de reproduction, ainsi que les taux de veaux nés et de veaux vivants d'une part pour les deux années en conduite normale et d'autre part pour les deux années avec traitement de superovulation.

Le taux cumulé de vaches fécondées pendant une campagne de reproduction varie de 80 à Ioo p. Ioo (groupe 2 VS groupe 3) pour les vélages 3 et4(différences non significatives).

La productivité numérique (nombre de veaux vivants pour Ioo vaches mises en reproduction) varie de 80 à 96 p. Ioo (groupe 2 VS groupe 6) en conduite normale et de 74 à III p. Ioo (groupe 9 VS groupe 3) après traitement de superovulation.

Le taux de mortinatalité varie en conduite normale de o à I 5,4 p. Ioo (groupes 2 ou 9 VS groupe I) et de 8,2 p. Ioo à 29,5 p. Ioo (groupe 3 VS groupe 5)après traitement de superovulation (différences non significatives). Ce taux élevé est expliqué par les conditions de vélage (assistance et environnement sanitaire).

\section{Discussion}

\section{I. - Nombre d'ovulations}

Il avait été montré précédemment qu'il existait des différences entre races de bovins quant à la sensibilité au traitement de superovulation à base de PMSG : Charolais VS FPN (MAuleon et al., I970), Angus, Hereford et Croisés (Turman 
et al., I97I), Angus VS Hereford (LAmond, I972), Charolais, Blondes d'Aquitaine et Croisées (Menissier et al., I974).

Les résultats présentés ici montrent (tabl. 2) que l'on observe chez les bovins des différences intra-race (entre groupes de demi-sœurs) sur le même critère. On retrouve ainsi des résultats comparables à ceux obtenus dans d'autres espèces notamment chez la souris (LAND et FALCONER, I969; BRADFORD, I968) chez la ratte (MARIANA, I974) et chez la brebis (BINDON, CH'ANG et TuRnER, I97I).

La relation entre le taux d'ovulation naturel et le taux d'ovulation induit semble différente selon les espèces. Chez la souris, ces deux réponses seraient indépendantes (LAND et FALCONER, I969). Chez la brebis au contraire, elles seraient liées puisque un troupeau sélectionné pour sa prolificité élevée en conduite normale

\section{TABLEAU 6}

Durée moyenne des cycles cestriens entre la puberté et la première campagne de reproduction et intervalle PMSG-cestrus lors des traitements de superovulation

Mean length of oestrus cycles between puberty and first breeding period and PMSG-oestrus interval during superovulation treatments

\begin{tabular}{|c|c|c|c|}
\hline $\begin{array}{l}\text { Groupes } \\
\text { (Groups) }\end{array}$ & $\begin{array}{l}\text { Durée moyenne des } \\
\text { cycles (j) (étendue) } \\
\text { (Mean length of } \\
\text { cycles }(d))\end{array}$ & $\begin{array}{c}\text { Pourcentage de génisses } \\
\text { ayant I ou plusieurs } \\
\text { cycles d'une durée } \\
<\mathrm{r} 8 \mathrm{j} \\
\text { (Percentage of heifers } \\
\text { exhibiting } I \text { or several } \\
\text { cycles lasting }<\mathrm{I} 8 \text { days) }\end{array}$ & $\begin{array}{c}\text { Intervalle PMSG } \\
\text { oestrus (j) } \\
\text { (PMSG-cestrus } \\
\text { interval }(d))\end{array}$ \\
\hline $\mathrm{I}$ & $20,9 \quad(12-27)$ & I I , I & $5,17-1,75$ \\
\hline 2 & I6,9 (o8-26) & 73,6 & $2,60=1,85$ \\
\hline 3 & $19,3\left(I_{5}-22\right)$ & 25,0 & $3,17=1,85$ \\
\hline 5 & I9, I $(08-24)$ & $23, I$ & $3,57 \div 1,29$ \\
\hline 6 & $20,5(18-24)$ & 0,0 & $3,91 \pm 2,20$ \\
\hline 9 & $19,6(10-23)$ & I0, 5 & $3,3^{8 \div} \mathrm{I}, 64$ \\
\hline
\end{tabular}

répond trois fois plus après le traitement d'induction que le troupeau sélectionné sur les naissances simples (BINDON, Ch'Ang et Turner, I97I). Chez les bovins ces données font défaut. On peut toutefois rapprocher les différences de nombre d'ovulations induites dans notre étude des différences de taux de naissances gémellaires observés en ferme. Toutefois, dans ce dernier cas, des différences de niveaux alimentaires, de système de conduite ou de saison peuvent expliquer une partie de la variation (BAR-ANAN et BOWMAN, I974) comme chez la brebis (REEves et RoBERTSON, I953). Dans notre expérience au contraire, tous les animaux sont conduits de la même façon pendant la durée de l'expérience. On peut remarquer d'ailleurs que l'on n'observe aucune naissance gémellaire pendant les deux années sans traitement.

Le nombre d'ovulation induit par un traitement hormonal peut-il être rattaché à d'autres critères de reproduction de la femelle? Chez la génisse Charolaise, l'âge au premier cestrus est variable. Pour les femelles considérées ici, le taux de génisses en œstrus à 15 mois variait de 7 I à 95 p. I0o: le groupe le moins précoce (groupe 2, 7 I p. IOo) est celui qui manifeste la plus forte réponse "nombre d'ovulation ", alors que le groupe le plus précoce (groupe 3,95 p. Ioo en œstrus à I 5 mois) est celui qui 
donne la plus faible réponse. Pour les autres groupes, nous n'avons pas observé de relation très définie ( $\mathrm{p}$. Ioo en ostrus à $\mathrm{I}_{5}$ mois $=8 \mathrm{I}$ p. Ioo, groupe $\mathrm{I}, 76 \mathrm{p}$. Ioo, groupe 5.8I p. Ioo, groupe 6 et 90 p. Ioo, groupe 9 ).

Les durées moyennes de cycles pendant la période qui précède la première mise en reproduction sont notées dans le tableau 6 ainsi que les intervalles entre injection de PMSG et début de l'œstrus lors des deux années de traitement. On constate des variations importantes de durées de cycles entre les groupes et en particulier, le groupe 2 se caractérise par des cycles très courts (moyenne I6,9 j 73,6 p. Ioo des cycles d'une durée inférieure à I 8 jours). Les intervalles moyens PMSG-œstrus varient de même. On ne note pas de relation entre ce critère et le nombre d'ovulations comme cela avait été montré précédemment (Mariana et al., I970).

\section{2. - Taux de gestation - Nombre de veaux nés ou vivants}

Les résultats portés dans le tableau 2 indiquent que la taille de la portée n'évolue pas comme le nombre moyen d'ovulations: ainsi, le groupe 3 donne la plus faible réponse en nombre d'ovulations et la plus forte en nombre de veaux; inversement, le groupe 2 donne la plus forte réponse en nombre d'ovulations et une réponse très moyenne en nombre de veaux.

\section{3. - Efficacité de la superovulation}

Nous avons cherché à déterminer l'efficacité de la superovulation, définie comme le nombre de veaux nés ou vivants pour Ioo ovules pondus. On observe des différences importantes (de I 2 à 60 p. Ioo) mais qui ne semblent pas liées directement au nombre moyen d'ovulations. Si la plus faible efficacité est obtenue dans le groupe 2 à forte réponse "nombre d'ovulation", on observe pour les trois groupes à réponse " nombre d'ovulation » faible $(\mathrm{I}, 3$ et 6$)$ des rendements faibles $(23 \%)$ moyens ( 46 p. Ioo) et élevés ( 60 p. Ioo).

Nous avons déterminé (tabl. 4) l'influence du nombre d'ovulations sur l'efficacité de la superovulation. Elle est maximum pour 2 ou 3 ovulations et très faible au-dessus de ce nombre. Le tableau I montre que cela peut expliquer en grande partie les différences d'efficacité de la superovulation entre fạmilles. Le classement par ordre croissant de réponses supérieures ou égales à 4 ovulations et le classement par taux décroissant d'efficacité sont identiques.

Ainsi, il apparaît une dissociation entre nombre d'ovulations et taille de la portée par le biais de la survie embryonnaire et périnatale. Ce phénomène a été clairement mis en évidence chez la souris : une augmentation du nombre d'ovulations entraîne bien un accroissement de la taille de la portée mais également du taux de mortalité embryonnaire (Bowman et RoBerTs, I958); inversement d'ailleurs, chez la souris, une sélection tendant à une diminution de la taille de la portée agit par une diminution de la survie embryonnaire, sans modification du taux d'ovulations (FALCONER, I960; BATEMAN, I966; Bradford, I968). Chez les ovins, un troupeau sélectionné sur les naissances gémellaires est supérieur au troupeau "naissance simple " non seulement en ce qui concerne le nombre d'ovulations, mais également pour le nombre d'embryons qui dégénèrent et la perte totale en embryons (Bindon, Ch'ang et Turner, r97i). Testart, Bosc et du Mesnil du Buisson (I968) ont montré une relation entre le nombre d'embryons par corne utérine et le taux de mortalité embryonnaire. Des résultats semblables ont été rapportés 
par VANDEPLASSChe (I968). Mariana et al. (I970) concluaient à l'existence d'un nombre optimum d'ovulations entre 2 et 4 en race FFPN. Les résultats présentés ici indiquent que cet optimum doit être comparable en race Charolaise. Dans la race Normande, et en inséminant avec un taureau de race différente (fœetus croisés) il semble que cet optimum soit plus élevé (5 à 6 ovulations). Ces résultats, ainsi, que la comparaison des groupes I, 3 et 6 de notre étude montrent bien qu'il existe des différences génétiques dans l'aptitude à mener à terme des gestations gémellaires.

\title{
4. - Bilan des vélages — Productivité numérique
}

Le taux de conception à chaque œstrus et la survie embryonnaire sont plus importants pour le bilan final que le nombre d'ovulations. Il apparaît souhaitable de contenir cette réponse ovarienne dans la limite de 2 à 4 ovulations : les groupes à réponses fortes (5 et 9) ont un bilan de vélage négatif comparé aux deux années en conduite normale.

Nous avons montré précédemment (Mariana et al., I970) qu'il était impossible d'obtenir régulièrement une réponse ovarienne homogène, même sur un lot de femelles homogènes au point de vue âge ou état sanitaire et nutritionnel. Les résultats présentés ici montrent que cela doit être possible par un choix des origines génétiques.

La deuxième conclusion que l'on peut tirer de l'étude présentée ici est que l'aptitude à mener à terme une gestation multiple est variable selon les lignées de demi-sœurs. Une sélection sur ce critère semble plus prometteuse que sur le nombre d'ovulations. LAMOND et GADDY (I972) émettent l'hypothèse que le taux de progestérone dans le sang périphérique serait plus faible pour un même nombre d'ovulations pour les vaches qui mèneraient à terme leur gestation. Si ce fait était vérifié, il y aurait un moyen simple de trier les vaches en vue de sélectionner celles dont l'utérus est le plus apte à accueillir plusieurs fœetus.

La combinaison d'une réponse ovarienne modérée et d'une survie embryonnaire élevée telle que nous l'observons dans notre groupe 3 nous a permis d'obtenir $x$ I I veaux vivants pour Ioo vaches mises en traitement : Ce n'est pas nécessairement le résultat maximum pour cette lignée, et l'effet de doses de PMSG plus élevées devra être étudié. De même, il sera nécessaire de vérifier sur un effectif plus important le bilan de reproduction de femelles du groupe 2 . Un troupeau de femelles des groupes 2 et 3 est en cours de constitution.

Accepté pour publication en avril 1977.

\section{Summary}

\author{
Within breed differences in the ovarian response \\ to superovulation treatment by PMSG in Charolais cows. \\ Frequency of twin birth
}

Six groups of half sisters of the Charolais breed have been tested upon their superovulation and twinning rates after injection of $x, 000$ IU of PMSG on the 16 th day of a normal oestrous cycle.

Supcrovulation rate varied from 32.5 to 75.0 per cent and mean number of ovulations from I.47 to 3.62. The efficiency of superovulation (number of calves born for Ioo ova) varied with 
the ovulation rate but also between groups (from 12 to $60 \%$ ). Part of this variation was due to differences in pregnancy rates between groups (from 31.5 to 66.6 for the same groups).

The number of calves born alive for yoo cows inseminated differed according to groups. Superovulation rate was not the main factor since the best calf crop (I I \%) was obtained with the low ovulation group ( 1.47 ova; group 3 ).

\section{Références bibliographiques}

Bar Anan R., Bowman J. C., 1974. Twinning Israeli Friesian dairy herds. Anim. Prod., 18, I O9-I 15 .

Bateman N., ig66. Ovulation and post ovulational losses in strains of mice selected from large and sma.ll litter. Genet. Res., 8, 229-242.

Bindon B. M., Ch'Ang T. S., Turner H. N., I97I. Ovarian response to gonadotrophin by merino ewes selected for fecondity. Aust. J. Agric. Res., 22 (5), 809-82o.

Bowman J. C., Roberts R. C., 1958. Embryonic mortality in relation to ovulation in the house mouse. J. Exp. Biol., 35, I $38-143$.

BRADFORD G. E., I968. Selection for litter size in mice in the presence and absence of gonadotrophin treatment. J. Antibiot., 21, $283-295$.

Falconer, I960. The genetic of litter size in mice. J. Cell. Comp. Physiol., 56 (suppl. I), I 53167.

Lamond D. R., 1972. Hormonal induction of multiple ovulations in the bovine. Proc. West. Sect. Amer. Soc. Anim. Sci., 23, 59I-595.

I.AMOND D. R., GADDY R. G., I972. Plasma progesterone in cows with multiple ovulations. J. Reprod. Fert., 29, 307-312.

Land R. B., Falconer D. S., i969. Genetic studies of ovulation rates in the mouse. Genet. lies. Camb., 13, 25-46.

Martana J. C., I969. Technique d'examen "in vivo " des ovaires chez la vache. Ann. Biol. anim. Bioch. Biophys., 9, 657-659.

Mariana J. C., Mauleon P., Benort M., Chupin D., i97o. Variabilité et répétabilité du nombre d'ovulations obtenues après injection de I 600 UI de PMSG et 1500 UI de HCG. Ann. Biol. anim. Bioch. Biophys., $\mathrm{n}^{\circ}$ hors série, FI, 47-63.

Mariana J. C., 1970. Étude sur le nombre d'ovulations obtenues après injection de PMSG chez la vache : notion de seuil de réponse. Ann. Biol. anim. Bioch. Biophys., 10, 575-579.

MARIANA J. C., I974. Étude du nombre d'ovulations après superovulation induite par PMSG chez trois souches de rattes adultes. Ann. Biol. anim. Bioch. Biophys., 14, 623-632.

Mauleon P., Mariana J. C., Benoit M., Solari A., Chupin D., ig7o. Influence de différentes doses de PMSG injectées en phase folliculaire du cycle cestrien sur le nombre et le rendement d'ovulations de vaches de race FlPN. Ann. Biol. anim. Bioch. Biophys., no hors série, FI, $3 I-46$.

Menissier F., Chupin D., Cheminnat E., Fabre F., Fabre G. P., 1974. Fertilité induite et sensibilité à la PMSG de femelles culardes. Ist World Congress on genetics applied to livestock production, $8 \mathrm{I}-98$.

Moore P. J., Greenwald G. S., I974. Seasonal variation in ovarian responsiveness of the cyclic hamster to PMSG. J. Reprod. Fert., 36, $219-220$.

Reeves E. C. R., Robertson F. W., I953. Factors affecting multiple births in sheep. Anim. Breed. Abstr., 21, 2I2-224.

Testart J., Bosc M. J., Du Mesnil du Butsson F., ig68. Étude de la survie embryonnaire après induction de superovulation chez la vache. VIe Cong. Intern. Reprod. Anim. Insem. Artif. Paris, vol. 1, 479-482.

Turman E. J., Laster D. B., Remberger R. E., Stephen D. F., ig7i. Multiple birth in beef cows treated with equine gonadotrophin (PMSG) and HCG. J. Anim. Sci., 32, 962-967.

VANDEPLASSCHE M., I968. La mortalité embryonnaire et son diagnostic. VIe Cong. Intern. Reprod. Anim. Insem. Artif. Paris, vol. 1, 347-391. 